\title{
Experimental Investigation on the Influence of Ambient Temperature on the Test Accuracy for the Differential Pressure Hydrostatic Levelling System
}

\author{
Li Bo, ${ }^{1}$ Chen Guo, ${ }^{1,2}$ and $W u$ Qi ${ }^{2}{ }^{2}$ \\ ${ }^{1}$ Advanced Road and Bridge Maintenance Engineering Technology Research Center of Jiangsu Province, \\ China Design Group Co.,Ltd., Nanjing 210000, China \\ ${ }^{2}$ Institute of Geotechnical Engineering, Nanjing Tech University, Nanjing 210009, China
}

Correspondence should be addressed to Wu Qi; qw09061801@163.com

Received 19 September 2021; Accepted 8 November 2021; Published 28 November 2021

Academic Editor: Lingkun Chen

Copyright (c) $2021 \mathrm{Li}$ Bo et al. This is an open access article distributed under the Creative Commons Attribution License, which permits unrestricted use, distribution, and reproduction in any medium, provided the original work is properly cited.

Hydrostatic levelling system (HLS) is widely used to monitor the settlement of major projects, such as high-speed railways, bridges, tunnels, dams, and nuclear power plants; ambient temperature is the most important influencing factor in the actual engineering settlement detection process. In order to systematically study the influence of ambient temperature $T_{A}$ on the test accuracy of the HLS, a test platform was built in the ambient temperature laboratory, and the influence of factors, including the amount of $T_{A}$ change, the rate of increase/decrease of $T_{A}$, the expansion coefficient of the connecting pipe, and the distance of the measuring point, on the HLS test accuracy was quantitatively analyzed. The test results show that the elevation of a single HSL case has a linear correlation with the ambient temperature; when the temperature rise rate is greater than $0.1^{\circ} \mathrm{C} / \mathrm{min}$, the measured data are distorted due to incomplete development of material expansion. The temperature influence coefficient of a single HSL case is linearly related to the expansion coefficient deviation between the refrigerant and pipe; the test error of the double HLS case caused by $T_{A}$ is attributed to the expansion coefficient deviation of the pipe and the refrigerant between the base station and the measuring point. The relative temperature influence coefficient increases as distance measurement increases, and the HLS test error caused by $T_{A}$ will maintain a constant value when the distance measurement exceeds a certain value.

\section{Introduction}

With the rapid socioeconomic development and urbanization, major national infrastructure projects, such as high-speed railways, large-span bridges, ultralong tunnels, high-rise buildings, integrated pipeline corridors, and dams, are increasing; their uneven settlement, deformation, and foundations are a precursor to the engineering failure $[1,2]$. Therefore, the accurate measurement for the settlement or deformation is crucial and can provide a scientific reference for the design, construction, and operation of major foundation projects; moreover, reliable data for numerical engineering analysis and long-term deformation prediction can be provided [3-5].

Settlement monitoring methods include geometric levelling, trigonometric levelling, GPS measurement, CCD optical imaging measurement, laser convergence measurement, hydrostatic levelling, and fibre grating measurement $[6,7]$. Among them, geometric levelling and trigonometric levelling are costly and inaccurate; besides, they are limited by topographical fluctuations and challenging to realize realtime monitoring $[7,8]$. The accuracy of GPS measurement for plane relative positioning has reached $0.1-1 \times 10^{-6}$, but its elevation measurement accuracy is only $10^{-2}$, which cannot meet some projects' accuracy requirements, and GPS is expensive [9]. Although the CCD optical imaging sensors, laser convergence sensors, and fibre grating sensors have the advantages of high accuracy, strong modality to the terrain, and a high degree of automation [10], the construction and maintenance costs are relatively high, and their anti-interference performance is poor [6]. Because of the simple 
structure, high accuracy, strong stability, and little influence from the external environment [11], hydrostatic levelling is widely used in the subsidence monitorization of major projects such as subways [12, 13], bridges [14], tunnels [11, 15-18], dams, and nuclear power plants [19].

The basic principle of the hydrostatic levelling system (HLS) is the liquid connector, which means that, by measuring the relative change of the liquid level in the levelling bowl at different positions, the settlement or uplift of each monitoring point relative to the base point can be determined [19]. However, the accuracy of the HLS is disturbed by factors, including temperature changes, pressure differences, earth tides, and gravity anomalies. [20]. Among them, the earth tides and gravity anomaly can only be filtered under constant temperature, and their influence can be ignored for nonprecision engineering because of the micron-level effect. The influence of pressure difference can be eliminated using equal-pressure pipes. In actual engineering, the temperature change is considered the most important influencing factor during settlement detection. Chen Jihua [21] thought that the uneven temperature would lead to uneven liquid density in the HLS, which leads to the test error; he also proposed a preliminary approach to eliminate temperature impact. Xu Shaofeng [22] investigated the influence of temperature and pressure change on the accuracy of HLS testing and proposed a temperature correction model considering the pipe's expansion coefficient. By installing a temperature sensor in the magnetostatic level bowl, Li Deqiao [9] analyzed the influence of temperature on HLS testing accuracy and verified the temperature correction model proposed by Xu Shaofeng. Based on field test data, Chen et al. [2] proposed a temperature gradient model, and it is suitable for the HLS test system.

In summary, there have been preliminary studies on temperature-induced HLS test errors, but it needs to be noted that most current research studies only focused on a single HLS case, and the temperature changes are concentrated in the order of $10^{-1}[17,18]$. However, during actual settlement monitoring, the annual ambient temperature basically varies from -10 to $30^{\circ} \mathrm{C}$. In addition, the existing temperature correction model, based on the water medium, no longer has practical engineering significance, especially in the subzero state; also, there are certain differences in the HLS measuring point spacing arrangement and pipe materials in the actual project.

In this paper, a differential pressure hydrostatic levelling platform was built in the ambient temperature laboratory to systematically study the impact of ambient temperature on HLS testing accuracy. The high-precision temperature sensor was used to collect the temperature data, the change of $T_{\mathrm{A}}$, the increase/decrease rate of $T_{\mathrm{A}}$, the expansion coefficient of the connecting pipe, and the distance of the measuring point on the HLS testing accuracy which is quantitatively analyzed. Furthermore, an evaluation method for the influence of ambient temperature on the HLS testing accuracy was developed.

\section{Test Platform Construction and Test Program}

2.1. Test Equipment and Materials. The Rosemount 3051S differential pressure hydrostatic levelling system was selected in this test because of the advantages of high sensitivity, wide frequency, lightweight, small size, and reliable operation, and its technical parameters are shown in Table 1. The platinum resistance temperature sensor was used to measure the ambient temperature. In order to ensure that the signals of the HLS and temperature sensor can be collected simultaneously and avoid time lag, a full-function collect instrument was selected. The closed-loop controlled ambient temperature chamber in the State Key Laboratory of Materials-Oriented Chemical Engineering of Nanjing Technology University was used to control the temperature changing rate and temperature difference. This equipment can precisely and linearly control the ambient temperature in the range of $-40-150^{\circ} \mathrm{C}$ with a fluctuation of less than $0.1^{\circ} \mathrm{C}$. The detailed performance parameters of the test chamber are shown in Table 2.

Because the liquid medium of "deionized water+ antifreeze" is likely to cause flocculation at low-temperature conditions and the thermal expansion coefficient of water increases with the increase of temperature, dimethicone was selected as the liquid medium in this test. The density of dimethicone is $1.03 \mathrm{~g} / \mathrm{cm}^{3}\left(20^{\circ} \mathrm{C}\right)$, and its effective operating temperature range is $-60-160^{\circ} \mathrm{C}$; the expansion coefficient in the operating temperature range is basically fixed at the value of $6.3 \times 10^{-4}$. To study the influence of the expansion coefficient of different connecting pipe materials on the HLS test accuracy, four kinds of connecting pipes with different expansion coefficients, including PU plastic pipe, polypropylene pipe, PTFE pipe, and 204 stainless steel pipe, were selected. The corresponding expansion coefficients are $3.35 \times 10^{-4}, 1.32 \times 10^{-4}, 1.45 \times 10^{-4}$, and $6.43 \times 10^{-5}$, respectively.

2.2. Test Platform Installation. Figure 1 shows the layout of the test platform. It can be seen that, in the ambient temperature test chamber, all HLS liquid pipes are connected, and one end is connected to the liquid storage tank; besides, all air pipes are connected, and one end is connected to the upper air section of the tank through a drying pipe. It should be pointed out that the pressure difference of a single HLS measuring point comes from the difference between the liquid pressure and the air pressure, which is an absolute value; the liquid/gas pressure difference between the HLS datum point and the test point is used to calculate the relative settlement between the measured point and the datum point, which is a relative value. The RT-1Q temperature sensor was arranged next to every HLS measuring point, and then the output signal of the HLS and temperature sensor can be collected by the VW-102E full-function reader. The technical parameters of the RT-1Q temperature sensor are shown in Table 3. 
TABLE 1: Technical parameters of the Rosemount 3051 S hydrostatic levelling system.

\begin{tabular}{lc}
\hline Index & Value \\
\hline Input voltage & $24 \mathrm{Vdc}$ \\
Input current & $200 \mathrm{~mA}$ \\
Power & $0.9 \mathrm{~W}$ \\
Output current (signal) & $4-20 \mathrm{~mA}$ \\
Effective pressure range & $0-60 \mathrm{kPa}$ \\
Effective operating temperature & $-50-65^{\circ} \mathrm{C}$ \\
\hline
\end{tabular}

TABle 2: Ambient temperature test chamber parameter performance index.

\begin{tabular}{lc}
\hline Index & Value \\
\hline Temperature range $\left({ }^{\circ} \mathrm{C}\right)$ & $-40-150$ \\
Temperature fluctuation $\left({ }^{\circ} \mathrm{C}\right)$ & $<0.1$ \\
Temperature rising rate $\left({ }^{\circ} \mathrm{C} / \mathrm{min}\right)$ & $<3-5$ \\
Temperature falling rate $\left({ }^{\circ} \mathrm{C} / \mathrm{min}\right)$ & $<0.7-1.5$ \\
Temperature sensor & Platinum resistance PT100 \\
\hline
\end{tabular}

2.3. Test Scheme. The influence of the ambient temperature on the test accuracy of the HLS usually can be reflected by the measurement error caused by temperature change. Considering the complex change of the annual ambient temperature of the detected structure, especially in winter and summer, two types of test platforms, including single HLS case (only single differential pressure hydrostatic levelling point) and double HLS cases (test base station + test point), were built in a laboratory with the standard ambient temperature to study the influence of the change of $T_{\mathrm{A}}$ $\left(-10^{\circ} \mathrm{C} \longrightarrow 30^{\circ} \mathrm{C} \longrightarrow-10^{\circ} \mathrm{C}\right)$ and the rate of increase/decrease of $T_{\mathrm{A}}$ on the accuracy of HLS test quantitatively. At the same time, combined with the actual engineering conditions and the environmental temperature change, the influence of the connecting pipe length between the base station and the test point (the distance between the test points) and the expansion coefficient of the connecting pipe (with obvious material differences) on the HLS test accuracy was comprehensively considered. The test conditions are shown in Table 4.

\section{Test Results and Analysis}

3.1. The Influence of the Ambient Temperature Rising/Falling Mode on the HLS Test Accuracy. In order to investigate the influence of the rising/falling mode of the ambient temperature on the HLS test accuracy, by converting the vertical pressure of the liquid medium, the settlement difference of case $\mathrm{C} 1$ caused by the temperature rising and falling was compared and analyzed. At the beginning of the test, the settlement of the HLS was recorded as zero for the convenience of analysis.

It can be seen from Figure 2 that, as ambient temperature $T_{A}$ linearly increased from $-10^{\circ} \mathrm{C}$ to $30^{\circ} \mathrm{C}$, the corresponding settlement $H_{E}$ of the HLS linearly decreased to $3.04 \mathrm{~mm}$; when $T_{A}$ linearly decreased from $30^{\circ} \mathrm{C}$ to $-10^{\circ} \mathrm{C}, H_{E}$ linearly rose from $3.04 \mathrm{~mm}$ to $0.03 \mathrm{~mm}$. The temperature influence coefficient $P_{t}=\Delta H_{E} / \Delta T_{A}$ was defined to quantitatively characterize the effect of ambient temperature on the HSL test accuracy; under the rising/falling mode of case $\mathrm{C} 1, P_{t}$ of the HLS was $-0.0774 \mathrm{~mm} /{ }^{\circ} \mathrm{C}$ and $-0.0731 \mathrm{~mm} /{ }^{\circ} \mathrm{C}$, respectively, and their relative deviation was $5.8 \%$, indicating that the rising or falling mode of ambient temperature had no significant effect on $P_{t}$.

\subsection{The Influence of Temperature Rising Rate on the HLS Test} Accuracy. Because these two ambient temperature changing modes have no significant effect on $P_{t}$, there is no need to conduct the falling mode test for cases $\mathrm{C} 2, \mathrm{C} 3$, and C4. Figure 3 shows the influence of the rising rate of temperature on the test accuracy of the single HSL. When the rising rate was 0.05 and $0.02^{\circ} \mathrm{C} / \mathrm{min}, H_{E}$ and $T_{A}$ of the HLS were strongly linearly correlated, and $P_{t}$ of cases $\mathrm{C} 1$ and $\mathrm{C} 4$ was $-0.0774 \mathrm{~mm} /{ }^{\circ} \mathrm{C}$ and $-0.0777 \mathrm{~mm} /{ }^{\circ} \mathrm{C}$, respectively. When the rising rate was $0.1^{\circ} \mathrm{C} / \mathrm{min}, H_{E}$ decreased as $T_{A}$ increased, but it needs to be noticed that the linear correlation between $T_{A}$ and $H_{E}$ was significantly weakened, and the data fluctuation became large. When the rising rate was $0.2^{\circ} \mathrm{C} / \mathrm{min}$, as $T_{A}$ linearly increased, $H_{E}$ increased with a decreasing rate. When $T_{A}>10^{\circ} \mathrm{C}, H_{E}$ nearly did not increase as $T_{A}$ increased; this is because the excessive rising rate of ambient temperature led to the incomplete expansion of materials and liquid media, resulting in data distortion and failing to reflect the impact of $T_{A}$ on the HLS test accuracy.

The relationship between $P_{t}$ and the temperature rising rate is shown in Figure 4. Combined with Figure 3, it can be seen that when the temperature rising rate was 0.02 or $0.05^{\circ} \mathrm{C} / \mathrm{min}$, the HLS worked normally, and $P_{t}$ was consistent. When the rising rate was greater than or equal to $0.1^{\circ} \mathrm{C} /$ min, the material expansion was not complete, and the data were distorted. In general, the temperature reached the lowest at 0 a.m., and the temperature reached the highest at 13:00. The maximum temperature difference during this period basically did not exceed $30^{\circ} \mathrm{C}$. Therefore, under natural conditions, the temperature changing rate was less than $0.04^{\circ} \mathrm{C} / \mathrm{min}$. Besides, considering the effectiveness of data collection and the test progress, the temperature changing rate was decided to be $0.05^{\circ} \mathrm{C} / \mathrm{min}$ for other test cases.

3.3. The Influence of Pipe Expansion Coefficient on the HLS Test Accuracy. In order to investigate the influence of the pipe expansion coefficient $\gamma_{\mathrm{pi}}$ on the single HSL test accuracy, a series of single-point test platforms were built with the PU plastic pipe, PTFE pipe, polypropylene pipe, and 204 stainless steel pipe, respectively. The test was carried out under a temperature rising rate of $0.05^{\circ} \mathrm{C} / \mathrm{min}$. As shown in Figure $5, H_{E}$ decreased linearly with the increase of $T_{A}$ for PU plastic pipes, while $H_{E}$ increased linearly with the increase of $T_{A}$ for PTFE pipes, polypropylene pipes, or 204 stainless steel pipes. This is because $\gamma_{\mathrm{pi}}$ of PU plastic pipes is greater than that of dimethicone, and the liquid level of simethicone decreased with the increase of $T_{A}$, leading to a decrease in elevation; by contrast, for PTFE pipes, polypropylene pipes, and 204 stainless steel pipes, $\gamma_{\mathrm{pi}}$ is obviously 


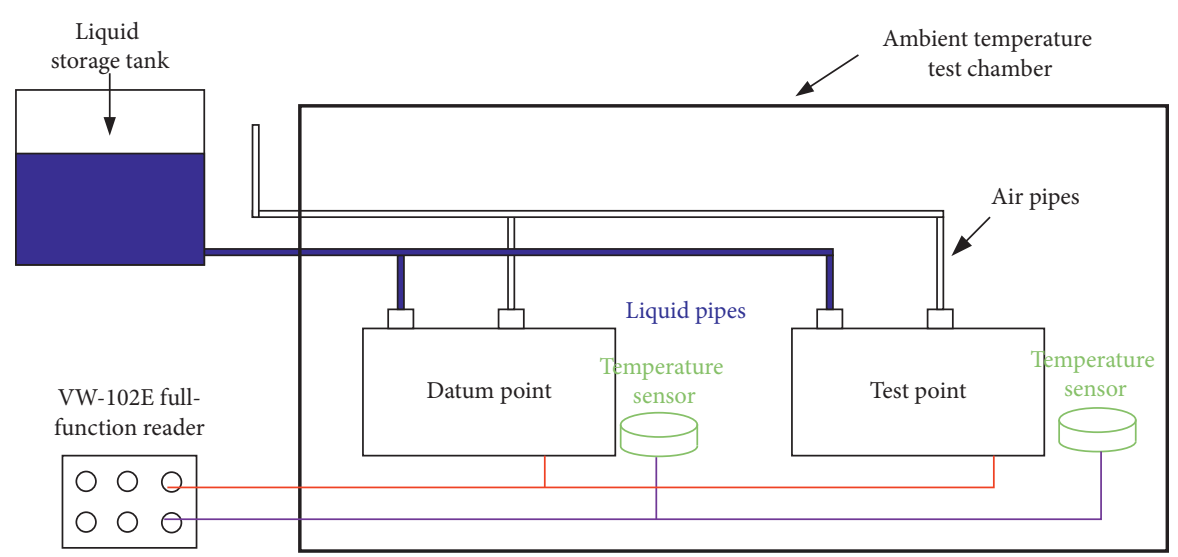

Figure 1: Schematic diagram of the working layout for the typical hydrostatic levelling system.

TABLE 3: Technical parameters of the RT-1Q temperature sensor.

\begin{tabular}{lc}
\hline Index & Value \\
\hline Effective operating temperature & $-40-80^{\circ} \mathrm{C}$ \\
Level of sensitivity & $\pm 0.005^{\circ} \mathrm{C}$ \\
Temperature measurement accuracy & $\pm 0.01^{\circ} \mathrm{C}$ \\
Water pressure resistance & $\geq 0.5 \mathrm{MPa}$ \\
Insulation resistance & $\geq 50 \mathrm{M} \Omega$ \\
Storage humidity & $\leq 80 \% \mathrm{RH}$ \\
Storage temperature & $-30-70^{\circ} \mathrm{C}$ \\
\hline
\end{tabular}

smaller than that of dimethicone, and the liquid level of simethicone rose with the increase of $T_{A}$, increasing $H_{E}$.

Furthermore, it was found that smaller $\gamma_{\mathrm{pi}}$ would not deliver the influence of $T_{A}$ on the single HSL measuring point. It is necessary to study the relationship between $P_{t}$ and the difference between the liquid medium expansion coefficient $\gamma_{\mathrm{di}}$ and the pipe expansion coefficient $\gamma_{\mathrm{pi}}$. As shown in Figure 6, $P_{t}$ increased with the increase of $\gamma_{\mathrm{di}^{-}} \gamma_{\mathrm{pi}}$, showing a linear relationship.

$$
P_{t}=0.04553\left(\gamma_{d i}-\gamma_{p i}\right)-0.0103
$$

It can be known that if $\gamma_{\mathrm{pi}}$ and $\gamma_{\mathrm{di}}$ are close, the influence of $T_{A}$ on the test accuracy of the single HSL measuring point can be ignored.

In order to study the effect of $\gamma_{\mathrm{pi}}$ on the double HSL test accuracy, the double HSL measuring point test platform was built with PU plastic pipes, PTFE pipes, and polypropylene pipes; HSL was used as a base station, and the other one was used as the measuring point. The test was carried out under the temperature raising rate of $0.05^{\circ} \mathrm{C} / \mathrm{min}$. Figure 7 shows the test data of the base station and the measuring point for case C8. It can be seen that $H_{E}$ of the base station and the measuring point increased with $T_{A}$, but the increasing rate of the elevation of the base station was apparently less than that of the measuring point, and $P_{t}$ presented a significant difference, which was $0.213 \mathrm{~mm} /{ }^{\circ} \mathrm{C}$ and $0.231 \mathrm{~mm} /{ }^{\circ} \mathrm{C}$, respectively.

Figure 8 (a) shows the relative elevation $\Delta H_{E}$ between the base station and the measuring point caused by $T_{A}$ 's increasing for case $\mathrm{C} 8$. The relative temperature influence coefficient $\Delta P_{t}$ is defined as the difference in $P_{t}$ between the base station and the measuring point. For case $C 8, \Delta P_{t}$ was $0.018 \mathrm{~mm} /{ }^{\circ} \mathrm{C}$, which is significantly smaller than that of C1-C7, suggesting that the measurement error caused by $T_{A}$ on the "liquid medium box-connecting pipe-base station" did not affect the relative elevation between the base station and the measuring point. The elevation difference was caused by the expansion difference between the connecting pipe and the refrigerant that connects the base station and the measuring point. Figures $8(\mathrm{~b})$ and $8(\mathrm{c})$ show $\Delta H_{E}$ between the base station and the measuring point for cases $\mathrm{C} 9$ and $\mathrm{C} 10$, which was caused by the increase of $T_{A}$. Similar to the single test point results, for polypropylene pipes, $\Delta H_{E}$ increased with $T_{A}$ but decreased for PU plastic pipes. However, similar to case $\mathrm{C} 8, \Delta P_{t}$ of cases $\mathrm{C} 9$ and $\mathrm{C} 10$ was also obviously smaller than that of the single-point test, and when the connecting pipe was a $\mathrm{PU}$ plastic, $\Delta P_{t}$ was negative. It should be pointed out that although the connecting pipe material had a significant effect on $\Delta P_{t}$, it was related to the length of the connecting pipe between the base station and the measuring point combined with the test results of cases C1-C9.

3.4. The Effect of Measuring Distance on the HLS Test Accuracy. According to the analysis above, when the connecting pipe material was fixed, the test error of the double or multiple HSL measurement points, which was induced by $T_{A}$ changing, was mainly determined by the distance of the measurement points. To systematically explore the effect of the distance between measurement points $D_{\text {test }}$ (the length of the connecting pipe) on the test accuracy of double HSL test points, PTFE pipes were used to construct the double measurement points' test platform, and the distance between the base station and the test point was set as $0.5 \mathrm{~m}, 1 \mathrm{~m}, 5 \mathrm{~m}$, and $10 \mathrm{~m}$. Figure 9 shows the relationship between $\Delta H_{E}$ and $T_{A}$. It can be seen that when $D_{\text {test }}$ increased to $1 \mathrm{~m}$ and $5 \mathrm{~m}$, $\Delta H_{E}$ also increased with the increase of $T_{A}$, but under the same $T_{A}$, the larger $D_{\text {test }}$ was, the larger $\Delta H_{E}$ was, which was consistent with the test results of case C8. Besides, $\Delta P_{t}$ of the double HLS test points was significantly smaller than $P_{t}$ of the base station under each working condition.

Figure 10 shows the relationship between $D_{\text {test }}$ and $\Delta P_{t} ; \Delta P_{t}$ increased with the increase of $D_{\text {test }}$. However, the increasing rate 
TABLE 4: The influence of ambient temperature on the measurement accuracy of the HLS.

\begin{tabular}{|c|c|c|c|c|c|c|}
\hline ID & $\begin{array}{c}\text { Test } \\
\text { platform }\end{array}$ & Pipe material & $\begin{array}{l}D_{\text {test }} \\
(\mathrm{m})\end{array}$ & $T_{\mathrm{A}}\left({ }^{\circ} \mathrm{C}\right)$ & $\begin{array}{l}T_{\mathrm{A}} \text { rate }\left({ }^{\circ} \mathrm{Cl}\right. \\
\min )\end{array}$ & Test purpose \\
\hline $\begin{array}{l}\mathrm{C} 1 \\
\mathrm{C} 2 \\
\mathrm{C} 3 \\
\mathrm{C} 4\end{array}$ & Single HLS & PU plastic pipe & - & $-10 \longrightarrow 30 \longrightarrow-10$ & $\begin{array}{c}0.05 \\
0.1 \\
0.2 \\
0.02\end{array}$ & $\begin{array}{c}\text { The effect of temperature rising/falling rate on the } \\
\text { HSL test error }\end{array}$ \\
\hline $\begin{array}{l}\mathrm{C} 5 \\
\mathrm{C} 6 \\
\mathrm{C} 7\end{array}$ & Single HLS & $\begin{array}{c}\text { PU plastic pipe } \\
204 \text { stainless steel } \\
\text { pipe } \\
\text { Polypropylene pipe }\end{array}$ & - & $-10 \longrightarrow 30$ & 0.05 & $\begin{array}{c}\text { The effect of pipe material on the single HSL test } \\
\text { error }\end{array}$ \\
\hline $\begin{array}{l}\mathrm{C} 8 \\
\mathrm{C} 9 \\
\mathrm{C} 10 \\
\end{array}$ & $\begin{array}{l}\text { Double } \\
\text { HLS }\end{array}$ & $\begin{array}{c}\text { PTFE pipe } \\
\text { Polypropylene pipe } \\
\text { PU plastic pipe }\end{array}$ & 0.5 & $-10 \longrightarrow 30$ & 0.05 & $\begin{array}{c}\text { The effect of pipe material on the double HSL test } \\
\text { error }\end{array}$ \\
\hline $\begin{array}{l}\text { C11 } \\
\text { C12 } \\
\text { C13 } \\
\end{array}$ & $\begin{array}{l}\text { Double } \\
\text { HLS }\end{array}$ & PTFE pipe & $\begin{array}{c}1 \\
5 \\
10 \\
\end{array}$ & $-10 \longrightarrow 30$ & 0.05 & $\begin{array}{l}\text { The effect of measuring distance on the single HSL } \\
\text { test error }\end{array}$ \\
\hline
\end{tabular}

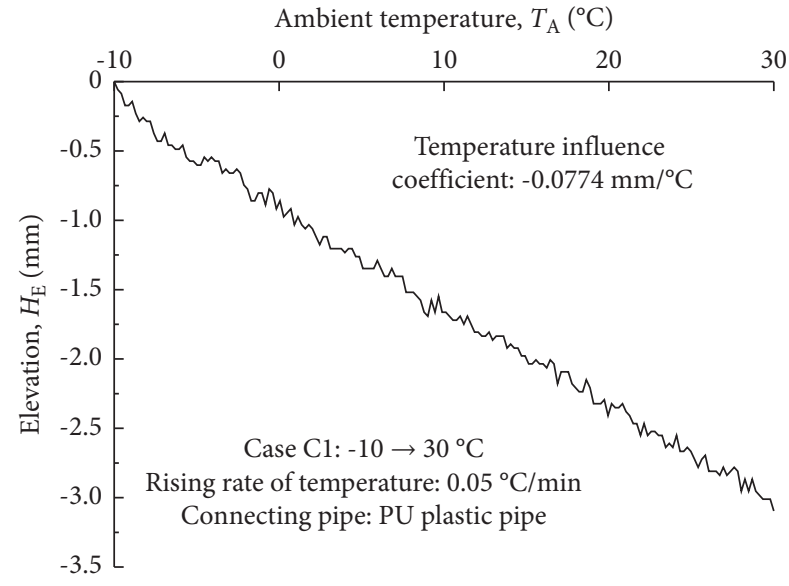

(a)

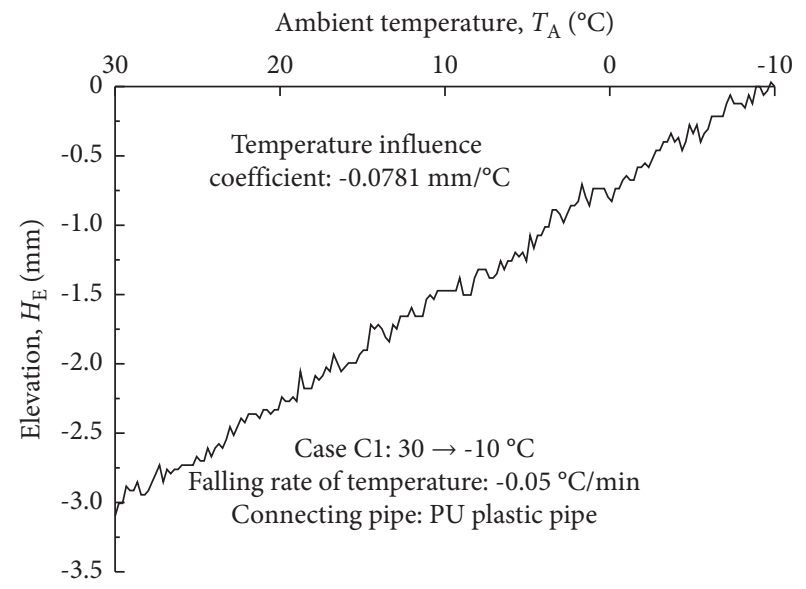

(b)

Figure 2: The influence of temperature rise/fall rate on the measurement accuracy of the HLS. (a) Ambient temperature rising. (b) Ambient temperature falling.

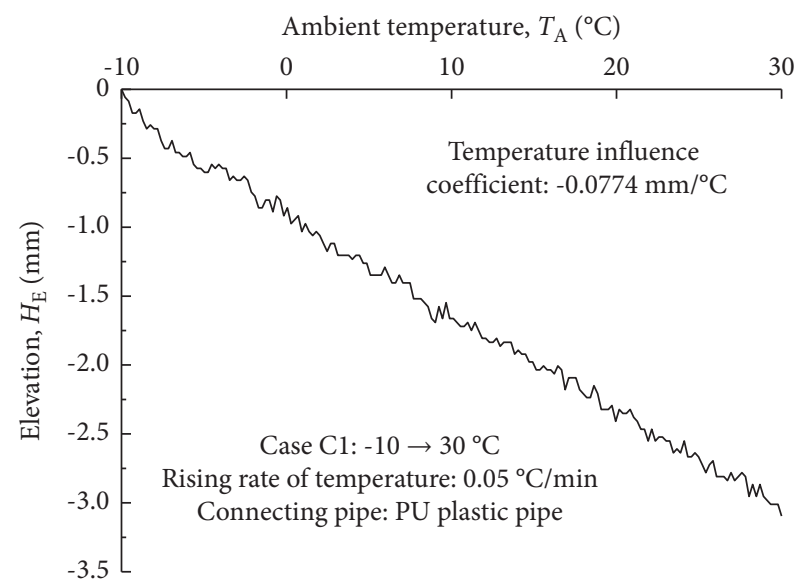

(a)

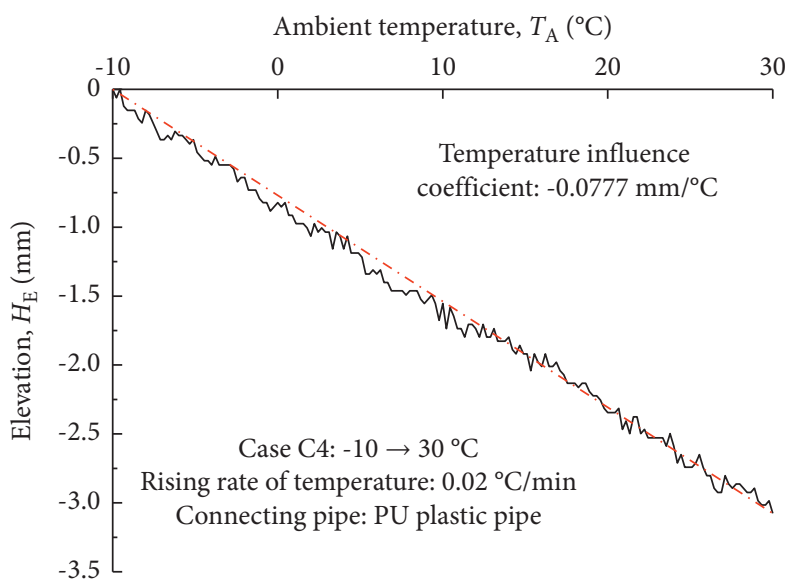

(b)

Figure 3: Continued. 


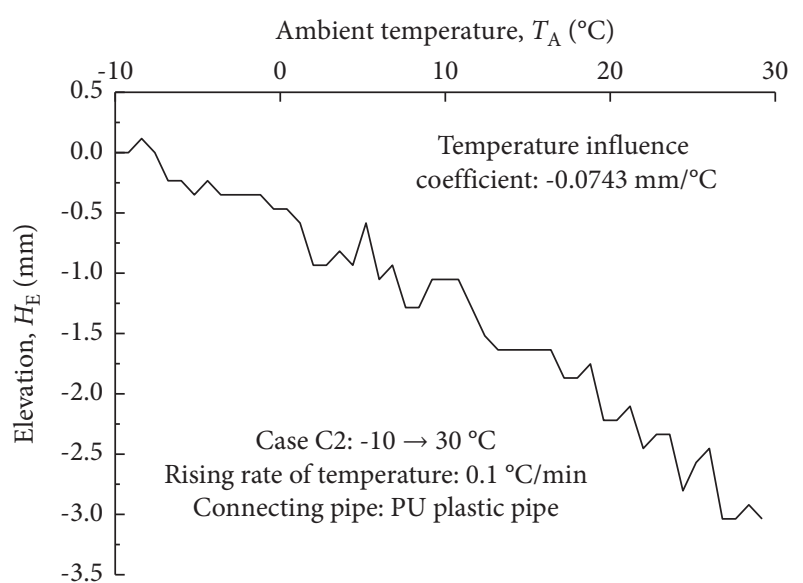

(c)

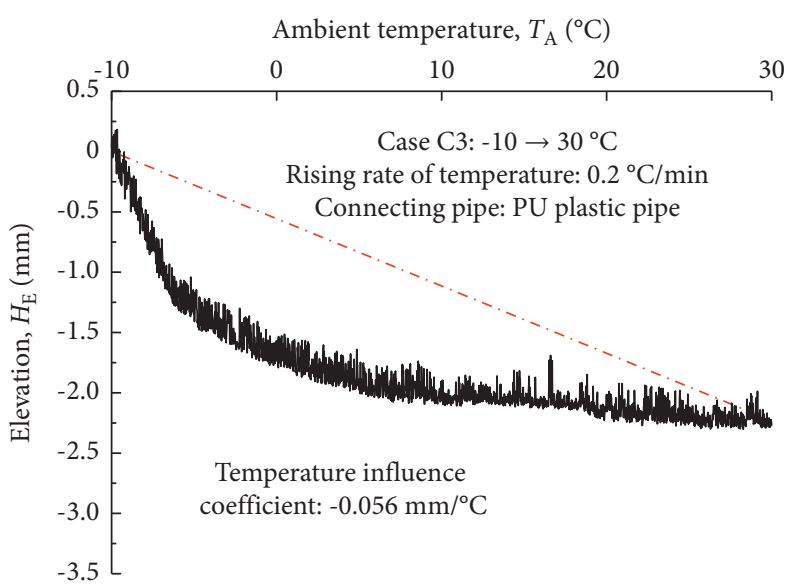

(d)

Figure 3: The influence of temperature rise rate on the measurement accuracy of the single HSL test point. (a) Case C1. (b) Case C4. (c) Case C2. (d) Case C3.

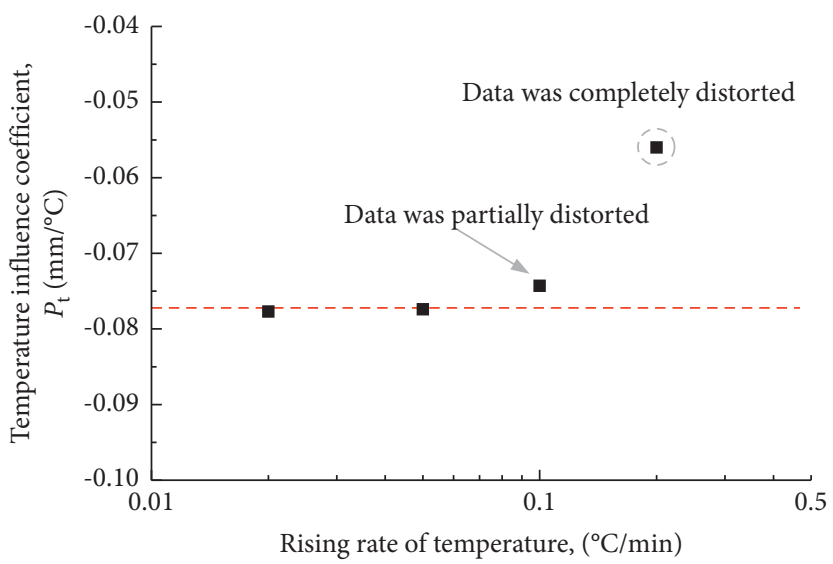

Figure 4: The relationship between temperature influence coefficient and temperature rise rate.

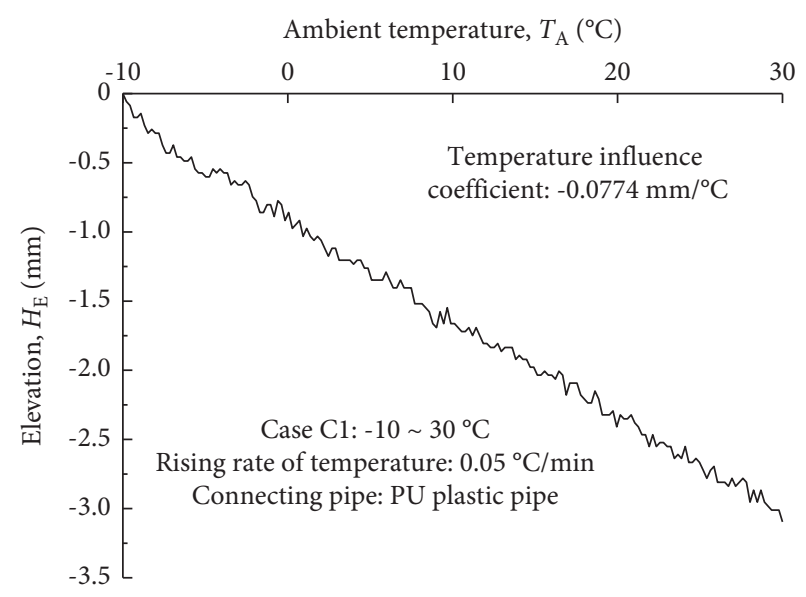

(a)

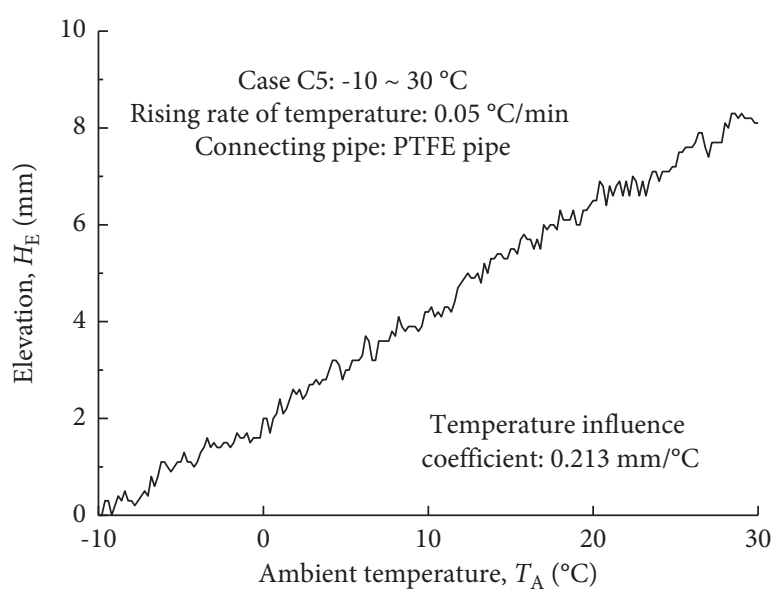

(b)

FIgURE 5: Continued. 


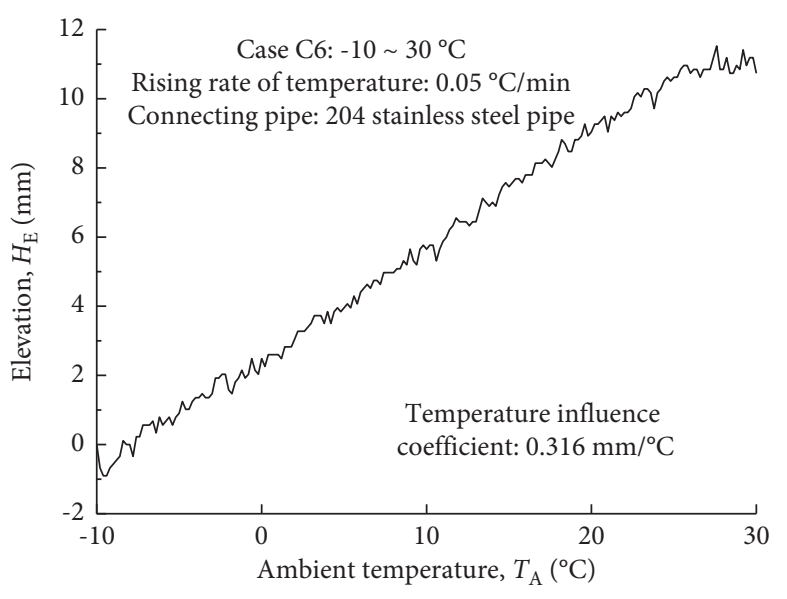

(c)

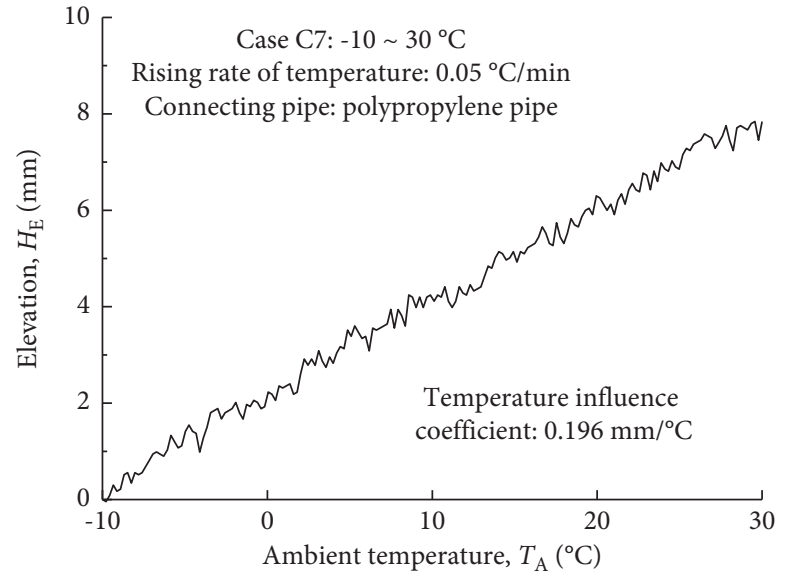

(d)

Figure 5: The influence of pipe expansion coefficient on the measurement accuracy of the single HSL test point. (a) PU plastic pipe. (b) PTFE pipe. (c) 204 stainless steel pipe. (d) Polypropylene pipe.

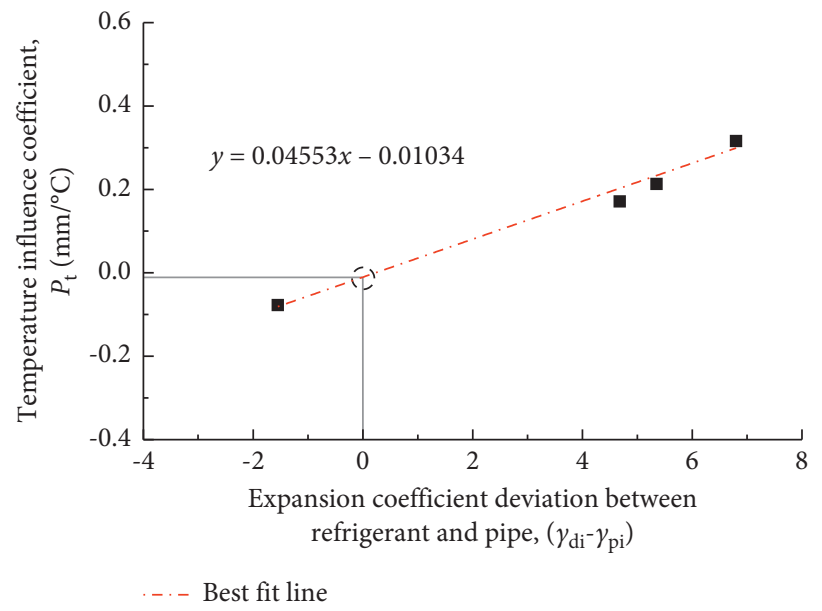

Figure 6: The relationship between the temperature influence coefficient and the expansion coefficient deviation between the refrigerant and pipe.

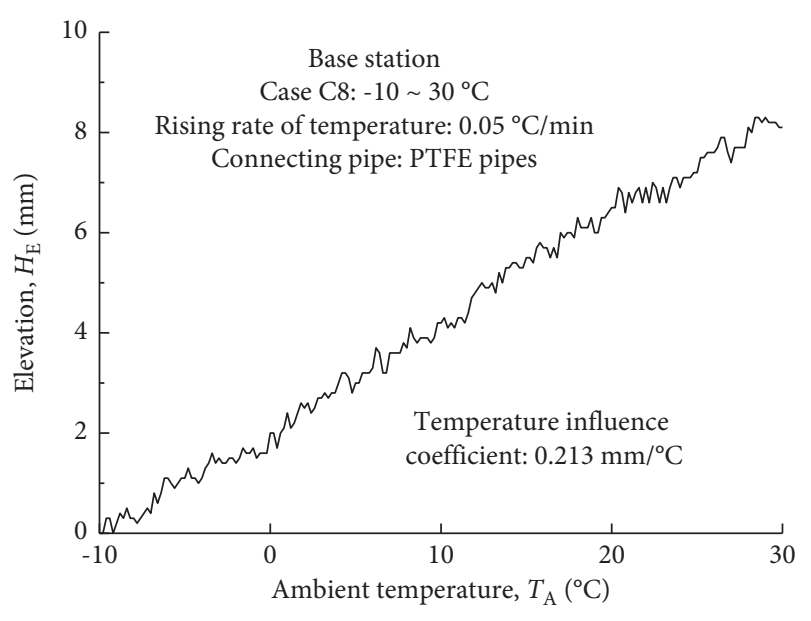

(a)

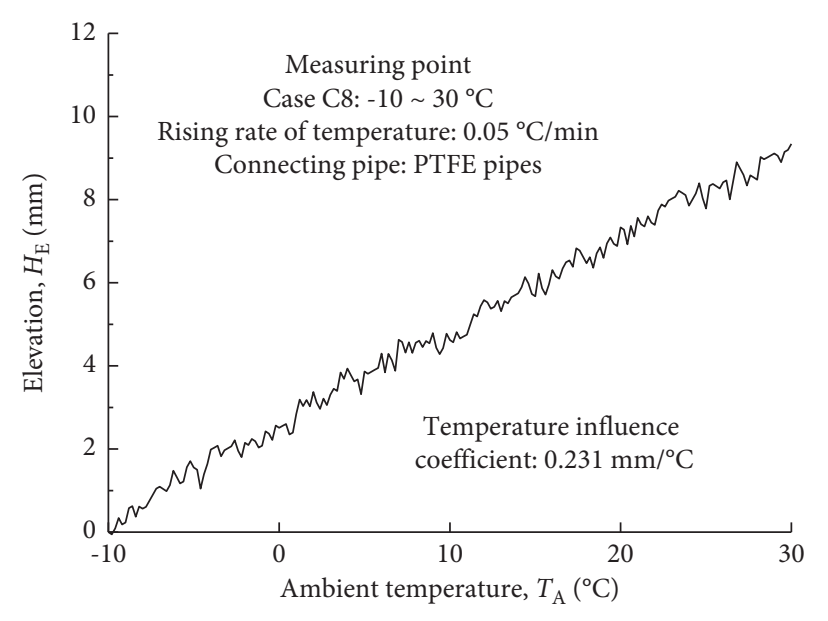

(b)

Figure 7: The relationship between ambient temperature and elevation of the base station and test point HSL for case C8. (a) Base station. (b) Measuring point. 


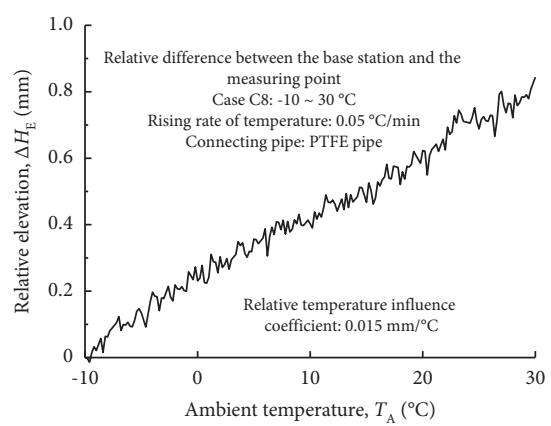

(a)

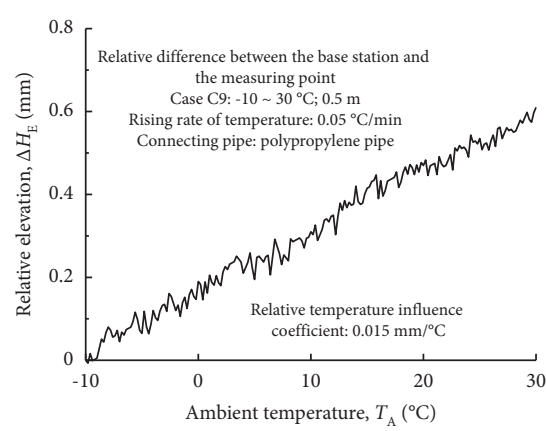

(b)

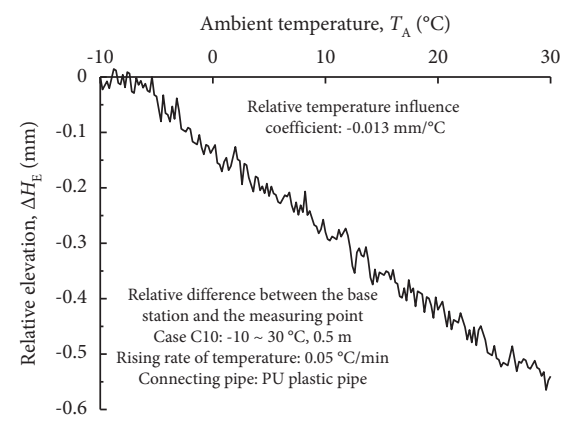

(c)

FIgURE 8: The relative elevation between the base station and test point HLS versus ambient temperature for cases C8-C10. (a) PTFE pipe. (b) Polypropylene 204 pipe. (c) PU plastic pipe.

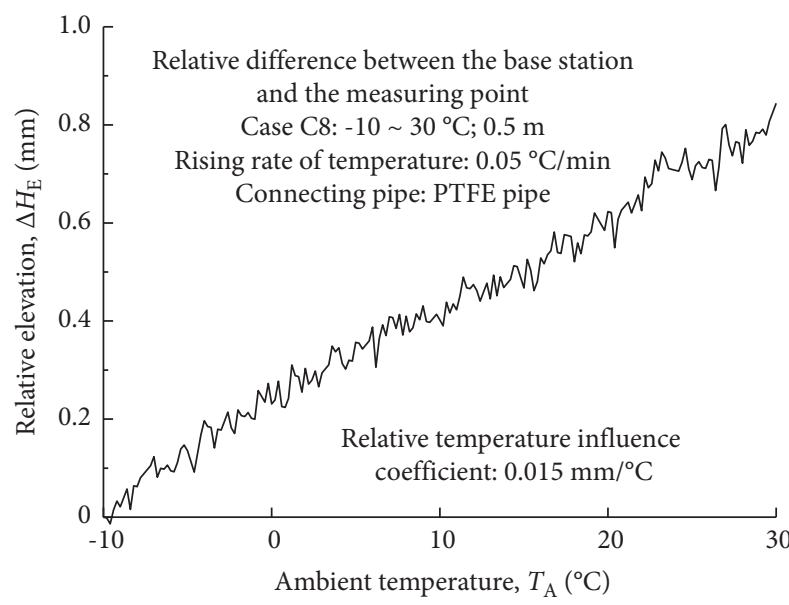

(a)

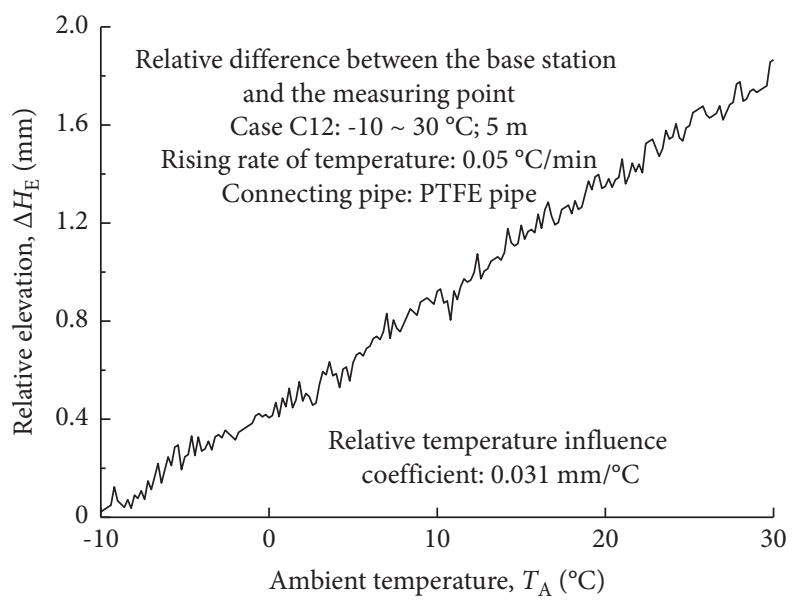

(c)

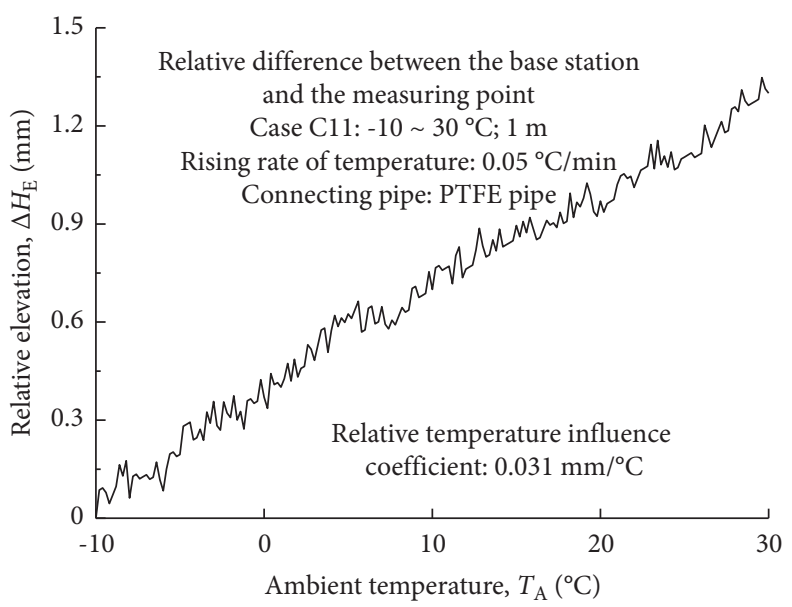

(b)

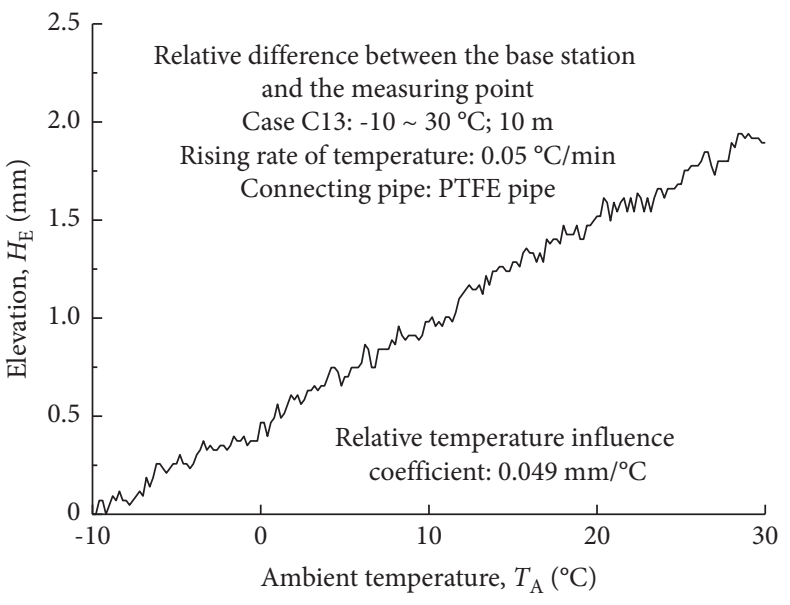

(d)

Figure 9: The relationship between relative elevation and ambient temperature under different measuring distances. (a) Case C8. (b) Case C11. (c) Case C12. (d) Case C13.

of $\Delta P t$ appears gradually decreased, and it can be inferred that when $D_{\text {test }}$ exceeds a certain value, $\Delta P_{t}$ may be maintained at a fixed value, but under larger $D_{\text {test }}$ conditions, the precise environmental temperature control is difficult to be achieved due to the limited test conditions. $D_{\text {test }}$ and $\Delta P_{t}$ present a power function relationship as follows: 


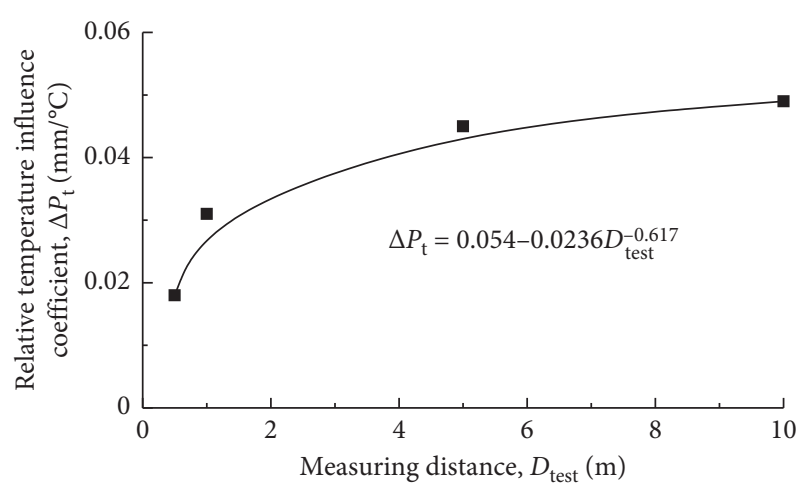

Figure 10: The relationship between measuring distance and relative temperature influence coefficient.

$$
\Delta P_{t}=0.054-0.0236 D_{\text {test }}^{-0.617} \text {. }
$$

Incorporating with the definition of $\Delta P_{t}$, under the ambient temperature considering the influence of $D_{\text {test }}$, the ambient temperature evaluation model of the HLS measuring points can be established:

$$
\begin{aligned}
\text { Set }_{\text {true }} & =\text { Set }_{\text {test }}-\Delta H_{E}, \\
\Delta H_{E} & =\left(0.054-0.0236 D_{\text {test }}^{-0.617}\right) \times \Delta T_{E},
\end{aligned}
$$

where Set $_{\text {true }}$ is the actual settlement of each HLS measuring point to eliminate the influence of temperature effect and Set $_{\text {test }}$ is the test settlement of each HLS measuring point.

\section{Conclusions and Discussion}

In this paper, a test platform with differential pressure hydrostatic levelling was installed in the ambient temperature laboratory. The high-precision temperature sensors were adopted to collect the temperature of each measurement point of the HLS, and the influence of factors, such as the ambient temperature, the increasing/decreasing rate of the ambient temperature, the expansion coefficient of the connecting pipe, and the distance of the measuring point, on the HLS test accuracy was quantitatively analyzed. The relevant conclusions can be drawn as follows:

(1) The elevation of the single HSL measuring point showed a single correlation with the ambient temperature. When the ambient temperature increasing rate was 0.02 or $0.05^{\circ} \mathrm{C} / \mathrm{min}$, the ambient temperature influence coefficient was consistent, and the test results were valid. When the increasing rate was greater than or equal to $0.1^{\circ} \mathrm{C} / \mathrm{min}$, the material expansion was not complete, and the data were distorted.

(2) The single HSL measuring point's temperature influence coefficient was linearly related to the difference in the expansion coefficient between the liquid medium and the pipe. When the expansion coefficient of the selected pipe was close to the expansion coefficient of the liquid medium, the effect of the temperature on single HSL test accuracy can be ignored.
(3) The test error of the double HLS measuring points caused by ambient temperature was attributed to the expansion difference between the connecting pipe and the liquid medium. The connecting pipe material and the length of the connecting pipe had a significant effect on the relative temperature influence coefficient.

(4) The relative temperature influence coefficient increased with the increase of the measurement distance, while the increase rate of the relative temperature influence coefficient gradually decreased, which preliminarily indicated that when the measurement distance exceeded a certain value, the HLS test error caused by the environmental temperature basically remains constant.

\section{Data Availability}

The data are generated from experiments and can be made available from the corresponding author upon request.

\section{Conflicts of Interest}

The authors declare that there are no conflicts of interest regarding the publication of this paper.

\section{Acknowledgments}

This work was supported by the National Science Foundation of China (52008206) and Institute of Crustal Dynamics, China Earthquake Administration (no. ZDJ201811).

\section{References}

[1] N. F. Li and Y. F. Zhao, "Application of hydrostatic leveling syste in XIANGJIABA hydropower station," Journal of Geodesy and Geodynamics, vol. 33, pp. 181-185, 2013.

[2] L. H. Chen, G. L. Guo, X. J. Zhu, and Q. Guo, "Precision analysis of deformation monitoring by hydrostatic level system," Safety In Coal Mines, vol. 46, no. 3, pp. 201-204, 2015.

[3] S. T. Liu and H. F. Zhang, "Research progress on deformation monitoring technology of surrounding rock of tunnel structure," Highway, vol. 9, pp. 262-268, 2013.

[4] H. Y. Zhuang, X. Yu, C. Zhu, and D. Jin, "Shaking table tests for the seismic response of a base-isolated structure with the SSI effect," Soil Dynamics and Earthquake Engineering, vol. 67, pp. 208-218, 2014.

[5] H. Zhuang, J. Fu, X. Yu, S. Chen, and X. Cai, "Earthquake responses of a base-isolated structure on a multi-layered soft soil foundation by using shaking table tests," Engineering Structures, vol. 179, no. 15, pp. 79-91, 2019.

[6] X. Wang, Y. Liu, and J. Yuan, "Development and application of settlement intelligent monitoring and evaluation system of high - speed railway," Journal of Railway Engineering Society, vol. 247, no. 4, pp. 20-25, 2019.

[7] J. Wei, P. Wei, and D. Q. Li, "Development and study on settlement remote monitoring and controlling system based on general packet radio service," China Civil Engineering Journal, vol. 48, no. S2, pp. 332-336, 2015.

[8] Z. L. Zhang, Y. Deng, C. L. Luo, and X. Hu, "Research on precise triangulated height surveying in place of first order 
leveling," Geomatics and Information Science of Wuhan University, vol. 31, no. 1, pp. 5-8, 2006.

[9] D. Q. Li, Studies on the Remote Settlement Monitoring System Based on Magnetostrictive Hydrostatic Leveling, Beijing JiaoTong University, China, Beijing, 2015.

[10] G. Y. Hou, Z. X. Li, T. Hu et al., "Study of tunnel settlement monitoring based on distributed optic fiber strain sensing technology," Rock and Soil Mechanics, vol. 41, no. 9, pp. 3148-3158, 2020.

[11] J. Jia and T. Zhang, "Application of high accuracy static level automatic system in bridge deformation monitoring," Highway Engineer, vol. 43, no. 2, pp. 172-176, 2018.

[12] F. Liu, M. D. He, L. Yu, and Y. Liu, "Measurement on the separation of monolithic roadbed from tunnel by hydrostatic leveling sensor," China Civil Engineering Journal, vol. 48, no. S2, pp. 356-360, 2015.

[13] C. P. Zhang, D. L. Zhang, J. J. Luo, and M. S. Wang, "Remote monitoring system applied to the construction of metro station undercrossing existing metro tunnel," Rock and Soil Mechanics, vol. 30, no. 6, pp. 1861-1866, 2009.

[14] Z. Liu, S. F. Zhang, and S. R. Cai, "Experimental study on deflection monitoring scheme of steep gradient and high drop bridge," Journal of Highway and Transportation Research and Development, vol. 32, no. 11, pp. 88-93, 2015.

[15] Z. G. Liu, H. W. Huang, Y. H. Zhao et al., "Immersed tube tunnel real -time health monitoring system," Hinese Journal of Underground Space and Engineering, vol. 4, no. 6, pp. 11101115, 2008.

[16] H. Q. Yang, J. C. Li, and Z. N. Chen, "Investigation on longitudinal ground subsidence caused by shallow tunnel excavation," Journal of Nanjing Tech University (Natural Science Edition), vol. 30, no. 3, pp. 56-60, 2008.

[17] W. Gang, X. J. Qin, D. Han, and Z. Liu, "Study on seepage and deformation characteristics of coal microstructure by $3 \mathrm{D}$ reconstruction of CT images at high temperatures," International Journal of Mining Science and Technology, vol. 31, no. 02, pp. 175-185, 2021.

[18] C. D. Su, J. D. Qiu, Q. H. Wu, and L. Weng, "Effects of high temperature on the microstructure and mechanical behavior of hard coal," International Journal of Mining Science and Technology, vol. 30, no. 05, pp. 643-650, 2020.

[19] Q. Zhang, X. Y. He, Z. Tang, and W. Wang, "Comparison of hydrostatic leveling system and wire position sensor for position monitoring in particle accelerator," Atomic Energy Science and Technology, vol. 51, no. 8, pp. 1532-1536, 2017.

[20] X. Y. He, "Application of hydrostatic leveling system in key scientific engineering and its developing tendency," Chinese Journal of Nuclear Science and Engineering, vol. 26, no. 4, pp. 332-336, 2006.

[21] J. H. Chen, "The influence of temperature unevenness on the accuracy of hydrostatic level," Geotechnical Invest igation \& Surveying.vol. 55, no. 1, pp. 51-57, 2000.

[22] S. F. Xu, Studies on Accuracy Theory and Validation of HLS Used in Particle Accelerators, University of science and Technology of China, Anhui, China, 2014. 